\title{
Analysis of Discontinuous Galerkin Approaches for Advection-Diffusion Problems
}

\author{
Loc Khieu* \\ Institute for Computational Science and Technology, Ho Chi Minh City, Vietnam \\ Krzysztof Fidkowski ${ }^{\dagger}$ and Eric Johnsen ${ }^{\ddagger}$ \\ The University of Michigan, Ann Arbor, MI 48109 USA
}

\begin{abstract}
A Discontinuous Galerkin discretization of $(3 p+1)$-order accuracy is presented for the advection-diffusion equations. For the diffusion term, the Recovery-based DG method is employed. For the advection term, the standard upwind DG discretization is replaced by an enhanced version with binary-recovery reconstruction. This reconstruction raises the order of accuracy, yet it preserves the compactness of the original stencil.
\end{abstract}

\section{Introduction}

Discontinuous Galerkin (DG) methods combine advantages of finite volume (FV) and finite element (FE) approaches into a single framework. The DG formulation, borrowed from the FE methodology, produces a high-order accurate and compact-stencil scheme with several advantages over high-order FV and finite difference (FD) methods, such as portability to complex geometries, scalability in parallel architectures and relatively simple extension to high order. The discontinuous nature of the basis functions in each cell introduces means for adding numerical dissipation in the convective terms, such that, unlike traditional FE schemes, convection-dominated flows can be captured in a stable fashion. The DG method was originally introduced for solving the steady-state neutron transport equation on triangular meshes ${ }^{[1]}$, and later analyzed ${ }^{[2-5]}$. The introduction of the Runge-Kutta DG (RKDG) method by Cockburn and Shu ${ }^{[6-9]}$ made this approach appealing for time-dependent convection-dominated problems. The RKDG method was shown to be well-suited to handle shocks through the use of slope limiters in one and two dimensions, on rectangular and triangular meshes. Similar to FV schemes, DG methods use Riemann solvers to determine the inter-cell flux and thus introduce the appropriate amount of dissipation at the discontinuities. They achieve a maximal order of accuracy $2 p+1$ in outputs.

However, the main difficulty preventing a simple extension to solving diffusive terms (i.e., second-order derivatives), such as in the Navier-Stokes equations, is the fact that derivatives are undefined at cell interfaces due to the discontinuity in the basis functions between neighboring cells. Two main approaches have been followed in the past: one in which gradient information is provided and which is stabilized by interior penalty terms or artificial diffusion with adjustable parameters;

${ }^{*}$ Researcher, Member AIAA, loc.h.khieu@gmail.com

${ }^{\dagger}$ Associate Professor, Aerospace Engineering, Member AIAA. kfid@umich.edu

$\ddagger$ Assistant Professor, Mechanical Engineering, Member AIAA. ejohnsen@umich.edu

Copyright (c) 2015 by Loc Khieu, Krzysztof Fidkowski, and Eric Johnsen. Published by the American Institute of Aeronautics and Astronautics, Inc. with permission. 

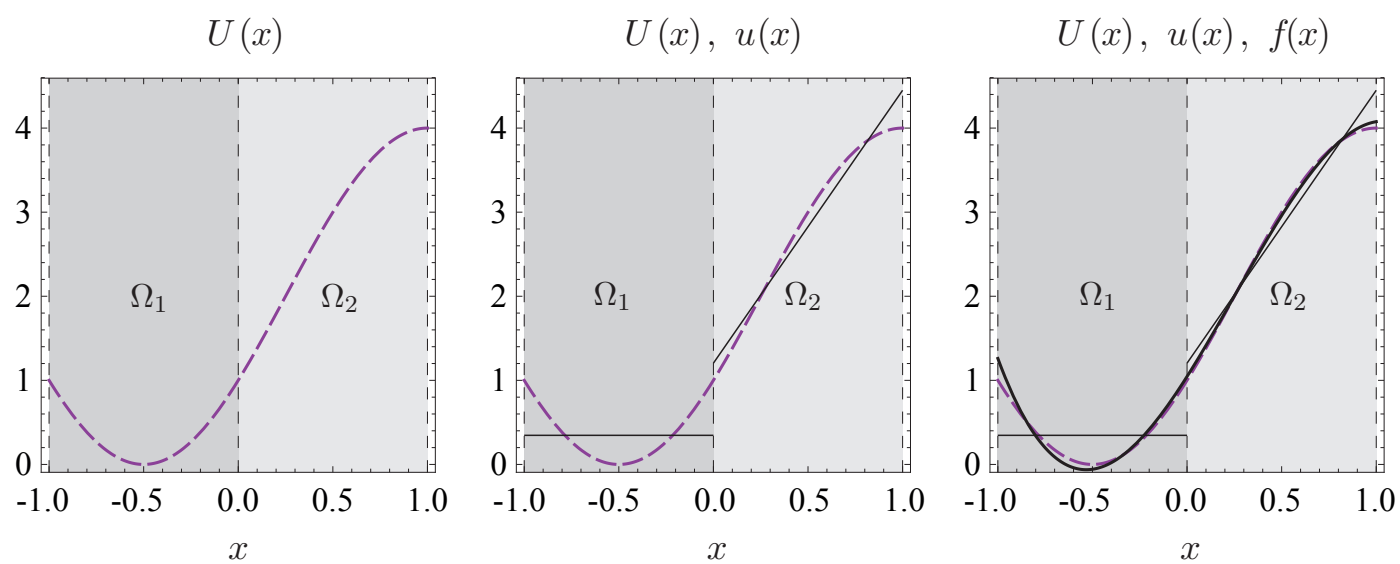

Figure 1: Recovery in one dimension for a piecewise-linear discretization $(p=1)$ on two adjacent intervals $(-1,0)$ and $(0,1)$. Shown are, from left to right, the original $\sin (x)$ initial value $U(x)$ (dashed), its piecewise-linear projection $u(x)$ (thin and solid), together with $U(x)$, and the cubic recovered function $f(x)$ (thick and solid) together with $u(x)$ and $U(x)$. All three distributions yield the same value when taking their inner product with either test function on either interval, making them indistinguishable in the weak sense.

and another more recent based on the idea of recovery of the underlying function over neighboring cells. With the former, interior penalty methods have been used for elliptic and parabolic problems ${ }^{[10-12]}$. Another class of methods treat the second-order partial differential equations as a system of first-order equations ${ }^{[13-16]}$. Such methods have been used to solve for diffusive terms, but there is no consistent methodology and often-adjustable parameters are required.

The Recovery-based DG (RDG) method for diffusion was introduced by Van Leer in $2005^{[17]}$. The interface discontinuity is removed in the weak sense by a local reconstructed polynomial called the recovered function $f$, which is required to satisfy moments with the original solution in the elements that span the interface. It is then used to compute the necessary function values and derivatives at the interface. Utilizing a $p$-order elemental tensor-product polynomial basis, RDG has been demonstrated to be stable ${ }^{[18]}$ and to achieve order $3 p+2$ or $3 p+1$ for $p$ even or odd, respectively ${ }^{[18]}$, on a Cartesian grid. Huynh ${ }^{[19]}$ shows that RDG is the most accurate, and that it has the most favorable stability restriction, in a comparison study of all contemporary DG schemes for diffusion. The result is robust: it holds in any number of dimensions, for linear as well as nonlinear equations, with or without mixed derivatives. Lo ${ }^{[20,21]}$ was the first to show the optimal accuracy for a 2-D diffusion-shear operator and for the 2-D Navier-Stokes terms; Johnsen et al. [22-24] showed it for 3-D turbulence calculations.

Now there exists a significant disparity in the order of accuracy between the diffusion discretization with RDG and the advection discretization with the standard method, $3 p+2 / 3 p+1$ of the former compared to $2 p+1$ of the latter. Our previous study ${ }^{[24]}$ shows that the overall performance is indeed limited. We are then prompted to explore various approaches for improving the DG discretization for advection ${ }^{[24,25]}$, and our preference is the interface-centered binary reconstruction, denoted icb. It reaches a maximal order of accuracy $3 p+1$ while still preserving a compact computational stencil, a notable advantage over the $P_{N} P_{M}$ approach by Dumbser ${ }^{[27,28]}$, even though the latter can achieve a higher order of accuracy (up to $4 p+3$ ).

In this paper, we shall study a discretization for the advection-diffusion equation that combines the RDG and the icb discretizations. 


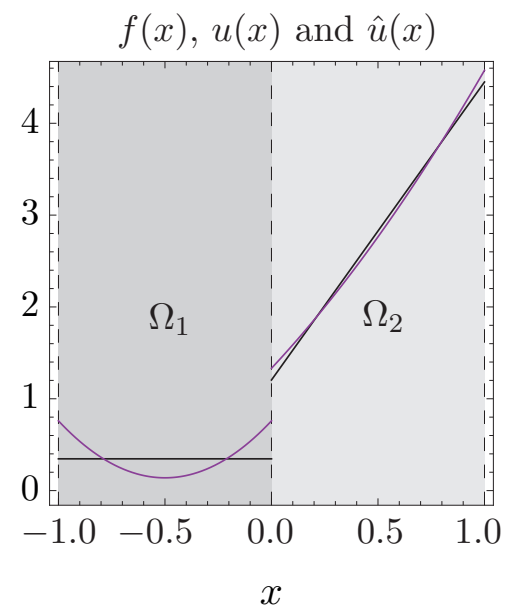

Figure 2: Illustration of the enhanced polynomials $\hat{u}_{L}$ and $\hat{u}_{R}$ for the icb1[0] scheme. The original discrete solutions $u_{1}(x)$ and $u_{2}(x)$ are carried over from Fig. 1.

\section{Recovery reconstruction}

To illustrate the principle of recovery reconstruction, it is sufficient to consider a problem of building a new polynomial from the original data in cells $\Omega_{1}$ and $\Omega_{2}$, see Fig. 1 . In the DG method, the numerical solutions $u_{1}(x)$ and $u_{2}(x)$ in those two cells are polynomials of degree $p$,

$$
u_{j}(x)=\sum_{\alpha=0}^{p} a_{j}^{(\alpha)} \phi_{j}^{(\alpha)}(x), \quad j=1,2,
$$

where $\phi_{j}^{(\alpha)}(x)$ are Legendre basis functions and $a_{j}^{(\alpha)}$ are the DG coefficients, or moments. The reconstructed polynomial, also called recovered function and denoted $f(x)$, is defined over the union of cells $\Omega_{1}$ and $\Omega_{2}$. It is required to preserve a certain number of moments of the original representations $u_{1}(x)$ and $u_{2}(x)$ in the weak sense. That means

$$
\begin{aligned}
\int_{\Omega_{1}} \phi_{1}^{(k)}(x) u_{1}(x) d x & =\int_{\Omega_{1}} \phi_{1}^{(k)}(x) f(x) d x, \quad k \in \mathcal{K}_{1}, \\
\int_{\Omega_{2}} \phi_{2}^{(k)}(x) u_{2}(x) d x & =\int_{\Omega_{2}} \phi_{2}^{(k)}(x) f(x) d x, \quad k \in \mathcal{K}_{2} .
\end{aligned}
$$

The sets $\mathcal{K}_{1}$ and $\mathcal{K}_{2}$ contains moments (values of indices $\alpha$ as in Eq. (1)) of $u_{1}(x)$ and $u_{2}(x)$, respectively, that $f$ has to preserve.

In the RDG discretization for diffusion, $\mathcal{K}_{1}=\mathcal{K}_{2}=[0, p]$, i.e., all available moments of $u_{1}(x)$ and $u_{2}(x)$ will be preserved. The recovered function $f$ is then a polynomial of degree $2 p+1$ that is continuous across the interface between $\Omega_{1}$ and $\Omega_{2}$ and indistinguishable, in the weak sense, from the original discontinuous discrete solutions. The function values and derivatives at the interface are calculated from $f$ and then used in appropriate terms of the diffusion discretization.

In the advection discretization icb, we now reconstruct two biased polynomials $\hat{u}_{L}(x)$ and $\hat{u}_{R}(x)$ of a higher-degree $\hat{p}$, instead of just one unique polynomial as the recovered function $f(x)$ in RDG. The "Left" and "Right" refer to the sides of the cell interface at $x=0$. The left-biased polynomial $\hat{u}_{L}(x)$ will preserve all $(p+1)$ moments of $u_{1}(x)$ of the left cell, and a subset of moments of $u_{2}(x)$ of the right cell. In Eqs. (2) and (3), $\mathcal{K}_{1}=[0, p]$ and all possibilities of $\mathcal{K}_{2} \equiv \mathcal{K}$ are shown in Table 1 for $p \in[1,2]$. Similarly, the right-biased $\hat{u}_{R}(x)$ will preserve all $(p+1)$ moments of $u_{2}(x)$, $\mathcal{K}_{2}=[0, p]$, and only a subset of all moments of $u_{1}(x)\left(\mathcal{K}_{1} \equiv \mathcal{K}\right)$. The values of $\hat{u}_{L}(x)$ and $\hat{u}_{R}(x)$ at 
Table 1: All possible interface-centered binary reconstruction (icb) schemes for $p \in[1,3]$.

\begin{tabular}{|c|c|c|c|}
\hline \multirow{2}{*}{$\begin{array}{c}p \\
(\text { unmodified ooa })\end{array}$} & \multicolumn{3}{|c|}{ Binary Reconstruction } \\
\hline & $\hat{p}($ ooa $)$ & $\mathcal{K}$ & Scheme names \\
\hline \multirow[t]{2}{*}{$1(3)$} & $2(4)$ & $\{0\}$ & icb1 [0] \\
\hline & & $\{1\}$ & icb1 [1] \\
\hline \multirow[t]{6}{*}{$2(5)$} & $4(7)$ & $\{0,1\}$ & $i \operatorname{cb} 2[0,1]$ \\
\hline & & $\{0,2\}$ & icb2 $[0,2]$ \\
\hline & & $\{1,2\}$ & icb2 $[1,2]$ \\
\hline & $3(6)$ & $\{0\}$ & icb2 [0] \\
\hline & & $\{1\}$ & icb2 [1] \\
\hline & & $\{2\}$ & icb2 [2] \\
\hline \multirow[t]{14}{*}{$3(7)$} & $6(10)$ & $\{0,1,2\}$ & icb3 $[0,1,2]$ \\
\hline & & $\{0,1,3\}$ & icb3 $[0,1,3]$ \\
\hline & & $\{0,2,3\}$ & icb3 $[0,2,3]$ \\
\hline & & $\{1,2,3\}$ & icb3 $[1,2,3]$ \\
\hline & $5(9)$ & $\{0,1\}$ & $i \operatorname{cb} 3[0,1]$ \\
\hline & & $\{0,2\}$ & icb3 $[0,2]$ \\
\hline & & $\{0,3\}$ & icb3 $[0,3]$ \\
\hline & & $\{1,2\}$ & icb3 $[1,2]$ \\
\hline & & $\{1,3\}$ & icb3 $[1,3]$ \\
\hline & & $\{2,3\}$ & icb3 $[2,3]$ \\
\hline & $4(8)$ & $\{0\}$ & icb3 [0] \\
\hline & & $\{1\}$ & icb3 [1] \\
\hline & & $\{2\}$ & icb3 [2] \\
\hline & & $\{3\}$ & icb3 [3] \\
\hline
\end{tabular}

the interface are then used for calculating the flux. Fourier analysis on the scalar linear advection shows that resulted schemes are maximally $(3 p+1)$-order ${ }^{[25]}$, as shown in Table 1 . Schemes of this family are denoted by $i c b \# 1[\# 2]$ where $\# 1$ is the value of $p$ and $\# 2$ is the subset $\mathcal{K}$. A specific example of the enhanced polynomials obtained in icb1[0] scheme is shown in Fig. 2. See below for the expressions of the enhanced polynomials for $p=1$ and $2 ; p=3$ is not shown because there are too many variations as shown in Table 1.

\section{II.A. The expressions of $\hat{u}_{L}$ and $\hat{u}_{R}$ for the icb schemes at $p=1$}

The original discretized solutions are represented by Legendre polynomials,

$$
u_{1}(x)=\sum_{\alpha=0}^{p} a_{1}^{(\alpha)} \phi_{1}^{(\alpha)}(x) \quad \text { and } \quad u_{2}(x)=\sum_{\alpha=0}^{p} a_{2}^{(\alpha)} \phi_{2}^{(\alpha)}(x)
$$

Expressions of the Legendre basis functions $\phi(x)$ change from cell to cell. However, if we map an arbitrary segment $\left[x_{j-1 / 2}, x_{j+1 / 2}\right]$ to a reference cell with a local coordinate $\xi \in[0,1]$, the 
Table 2: The basis functions of the Legendre polynomial in the reference coordinate.

\begin{tabular}{l|l}
\hline$\phi(x)$ & \\
\hline$\phi^{(0)}(\xi)$ & 1 \\
$\phi^{(1)}(\xi)$ & $-1+2 \xi$ \\
$\phi^{(2)}(\xi)$ & $1-6 \xi+6 \xi^{2}$ \\
$\phi^{(3)}(\xi)$ & $-1+12 \xi-30 \xi^{2}+20 \xi^{3}$ \\
$\phi^{(4)}(\xi)$ & $1-20 \xi+90 \xi^{2}-140 \xi^{3}+70 \xi^{4}$ \\
$\phi^{(5)}(\xi)$ & $-1+30 \xi-210 \xi^{2}+560 \xi^{3}-630 \xi^{4}+252 \xi^{5}$ \\
$\phi^{(6)}(\xi)$ & $1-42 \xi+420 \xi^{2}-1680 \xi^{3}+3150 \xi^{4}-2772 \xi^{5}+924 \xi^{6}$ \\
$\phi^{(7)}(\xi)$ & $-1+56 \xi-756 \xi^{2}+4200 \xi^{3}-11550 \xi^{4}+16632 \xi^{5}-12012 \xi^{6}+3432 \xi^{7}$ \\
$\phi^{(8)}(\xi)$ & $1-72 \xi+1260 \xi^{2}-9240 \xi^{3}+34650 \xi^{4}-72072 \xi^{5}+84084 \xi^{6}-51480 \xi^{7}+12870 \xi^{8}$ \\
\hline
\end{tabular}

expressions for $\phi(\xi)$ are universal; see Table 2 for the expressions of $\phi(\xi)$ up to $p=8$. To get back $\phi(x)$, we apply the transformation $x=\Delta x_{j} \xi+x_{j-1 / 2}$. Note that they are orthogonal,

$$
\int_{x_{j-1 / 2}}^{x_{j+1 / 2}} \phi_{j}^{(\alpha)}(x) \phi_{j}^{(\beta)}(x) \neq 0 \text { only when } \alpha \neq \beta \text {. }
$$

The enhanced polynomials $\hat{u}_{L}$ and $\hat{u}_{R}$ are expressed by:

$$
\hat{u}_{L}=\sum_{\alpha=0}^{\hat{p}} \hat{a}_{L}^{(\alpha)} \phi_{1}^{(\alpha)}(x) \text { and } \hat{u}_{R}=\sum_{\alpha=0}^{\hat{p}} \hat{a}_{R}^{(\alpha)} \phi_{2}^{(\alpha)}(x) .
$$

Values of $\hat{a}_{L}^{(\alpha)}$ are solutions of the following system:

$$
\begin{aligned}
\int_{\Omega_{1}} \phi_{1}^{(k)}(x) u_{1}(x) d x & =\int_{\Omega_{1}} \phi_{1}^{(k)}(x) \hat{u}_{L} d x, \quad k \in[0, p], \\
\int_{\Omega_{2}} \phi_{2}^{(k)}(x) u_{2}(x) d x & =\int_{\Omega_{2}} \phi_{2}^{(k)}(x) \hat{u}_{L} d x, \quad k \in \mathcal{K} .
\end{aligned}
$$

Vice versa, the system to solve for $\hat{a}_{R}^{(\alpha)}$ is:

$$
\begin{aligned}
\int_{\Omega_{1}} \phi_{1}^{(k)}(x) u_{1}(x) d x & =\int_{\Omega_{1}} \phi_{1}^{(k)}(x) \hat{u}_{R} d x, \quad k \in \mathcal{K}, \\
\int_{\Omega_{2}} \phi_{2}^{(k)}(x) u_{2}(x) d x & =\int_{\Omega_{2}} \phi_{2}^{(k)}(x) \hat{u}_{R} d x, \quad k \in[0, p] .
\end{aligned}
$$

Owing to the orthogonality of the Legendre basis functions, $\hat{a}_{L}^{(\alpha)}=a_{1}^{(\alpha)}$ and $\hat{a}_{R}^{(\alpha)}=a_{2}^{(\alpha)}$ for all $\alpha \in[0, p]$.

For $p=1$, the expressions for the remaining coefficients are:

- icb1[0]

$$
\hat{a}_{L}^{(2)}=\frac{1}{6}\left(-a_{1}^{(0)}-2 a_{1}^{(1)}+a_{2}^{(0)}\right), \quad \hat{a}_{R}^{(2)}=\frac{1}{6}\left(-a_{2}^{(0)}+2 a_{2}^{(1)}+a_{1}^{(0)}\right) .
$$

- icb1[1]

$$
\hat{a}_{L}^{(2)}=\frac{1}{6}\left(-a_{1}^{(1)}+a_{2}^{(1)}\right), \quad \hat{a}_{R}^{(2)}=\frac{1}{6}\left(a_{2}^{(1)}-a_{1}^{(1)}\right) .
$$


II.B. The expressions of $\hat{u}_{L}$ and $\hat{u}_{R}$ for the icb schemes at $p=2$

For $p=2$,

- icb2 $[0,1]$ the remaining coefficients for $\hat{u}_{L}$

$$
\begin{aligned}
& \hat{a}_{L}^{(3)}=\frac{1}{256}\left(-73 a_{1}^{(0)}-101 a_{1}^{(1)}-168 a_{1}^{(2)}+73 a_{2}^{(0)}-45 a_{2}^{(1)}\right), \\
& \hat{a}_{L}^{(4)}=\frac{1}{256}\left(15 a_{1}^{(0)}+19 a_{1}^{(1)}+24 a_{1}^{(2)}-15 a_{2}^{(0)}+11 a_{2}^{(1)}\right) ;
\end{aligned}
$$

and for $\hat{u}_{R}$

$$
\begin{aligned}
& \hat{a}_{R}^{(3)}=\frac{1}{256}\left(73 a_{2}^{(0)}-101 a_{2}^{(1)}+168 a_{2}^{(2)}-73 a_{1}^{(0)}-45 a_{1}^{(1)}\right), \\
& \hat{a}_{R}^{(4)}=\frac{1}{256}\left(15 a_{2}^{(0)}-19 a_{2}^{(1)}+24 a_{2}^{(2)}-15 a_{1}^{(0)}-11 a_{1}^{(1)}\right) .
\end{aligned}
$$

- icb2 $[0,2]$ for the remaining coefficients of $\hat{u}_{L}(x)$ :

$$
\begin{aligned}
& \hat{a}_{L}^{(3)}=\frac{1}{64}\left(-7 a_{1}^{(0)}-14 a_{1}^{(1)}-33 a_{1}^{(2)}+7 a_{2}^{(0)}-9 a_{2}^{(2)}\right), \\
& \hat{a}_{L}^{(4)}=\frac{1}{320}\left(5 a_{1}^{(0)}+10 a_{1}^{(1)}+19 a_{1}^{(2)}-5 a_{2}^{(0)}+11 a_{2}^{(2)}\right) ;
\end{aligned}
$$

and for $\hat{u}_{R}(x)$ :

$$
\begin{aligned}
& \hat{a}_{R}^{(3)}=\frac{1}{64}\left(7 a_{2}^{(0)}-14 a_{2}^{(1)}+33 a_{2}^{(2)}-7 a_{1}^{(0)}+9 a_{1}^{(2)}\right), \\
& \hat{a}_{R}^{(4)}=\frac{1}{320}\left(5 a_{2}^{(0)}-10 a_{2}^{(1)}+19 a_{2}^{(2)}-5 a_{1}^{(0)}+11 a_{1}^{(2)}\right) .
\end{aligned}
$$

- icb2 $[1,2]$ for the remaining coefficients of $\hat{u}_{L}(x)$ :

$$
\begin{aligned}
& \hat{a}_{L}^{(3)}=\frac{1}{320}\left(-35 a_{1}^{(1)}-137 a_{1}^{(2)}+35 a_{2}^{(1)}-73 a_{2}^{(2)}\right), \\
& \hat{a}_{L}^{(4)}=\frac{1}{64}\left(a_{1}^{(1)}+3 a_{1}^{(2)}-a_{2}^{(1)}+3 a_{2}^{(2)}\right)
\end{aligned}
$$

and for $\hat{u}_{R}(x)$ :

$$
\begin{aligned}
& \hat{a}_{R}^{(3)}=\frac{1}{320}\left(-35 a_{2}^{(1)}+137 a_{2}^{(2)}+35 a_{1}^{(1)}+73 a_{1}^{(2)}\right), \\
& \hat{a}_{R}^{(4)}=\frac{1}{64}\left(-a_{2}^{(1)}+3 a_{2}^{(2)}+a_{1}^{(1)}+3 a_{1}^{(2)}\right) .
\end{aligned}
$$

- icb2 [0] for the remaining coefficients of $\hat{u}_{L}(x)$ :

$$
\hat{a}_{L}^{(3)}=\frac{1}{22}\left(-a_{1}^{(0)}-2 a_{1}^{(1)}-6 a_{1}^{(2)}+a_{2}^{(0)}\right)
$$

and for $\hat{u}_{R}(x)$ :

$$
\hat{a}_{R}^{(3)}=\frac{1}{22}\left(a_{2}^{(0)}-2 a_{2}^{(1)}+6 a_{2}^{(2)}-a_{1}^{(0)}\right) .
$$


- icb2 [1] for the remaining coefficients of $\hat{u}_{L}(x)$ :

$$
\hat{a}_{L}^{(3)}=\frac{1}{30}\left(-a_{1}^{(1)}-6 a_{1}^{(2)}+a_{2}^{(1)}\right) ;
$$

and for $\hat{u}_{R}(x)$ :

$$
\hat{a}_{R}^{(3)}=\frac{1}{30}\left(-a_{2}^{(1)}+6 a_{2}^{(2)}+a_{1}^{(1)}\right) .
$$

- icb2 [2] for the remaining coefficients of $\hat{u}_{L}(x)$ :

$$
\hat{a}_{L}^{(3)}=\frac{1}{10}\left(-a_{1}^{(2)}+a_{2}^{(2)}\right)
$$

and for $\hat{u}_{R}(x)$ :

$$
\hat{a}_{R}^{(3)}=\frac{1}{10}\left(a_{2}^{(2)}-a_{1}^{(2)}\right)
$$

\section{Results from Fourier Analysis}

\section{III.A. Methodology}

We perform the Fourier analysis of the DG scheme for the scalar linear advection-diffusion equation

$$
\partial_{t} u+a \partial_{x} u=\mu \partial_{x x} u
$$

where $\mu$ is coefficient of diffusion and $a$ is advection speed (assumed to be positive without any loss of generality). The DG update equations have the following general form:

$$
\begin{aligned}
\frac{d}{d t} \int_{\Omega_{j}} v_{j} u_{j} d x & =-a \int_{\Omega_{j}} v_{j} \partial_{x} u_{j} d x+\mu \int_{\Omega_{j}} v_{j} \partial_{x x} u_{j} d x \\
\Rightarrow \frac{d}{d t} \int_{\Omega_{j}} v_{j} u_{j} d x= & -a\left[v_{j} u_{j}\right]_{x_{j-1 / 2}}^{x_{j+1 / 2}}+a \int_{\Omega_{j}} u_{j} \partial_{x} v_{j} d x \\
& +\mu\left[v_{j} \partial_{x} u_{j}-u_{j} \partial_{x} v_{j}\right]_{x_{j-1 / 2}}^{x_{j+1 / 2}}+\mu \int_{\Omega_{j}} u_{j} \partial_{x x} v_{j} d x
\end{aligned}
$$

In evaluation of the advection boundary terms, $\left[v_{j} u_{j}\right]_{x_{j-1 / 2}}^{x_{j+1 / 2}}$, the interface values take the upwind direction,

$$
\begin{aligned}
& u_{j-1 / 2}=u\left(\hat{u}_{j-1 / 2, L}\left(x_{j-1 / 2}\right), \hat{u}_{j-1 / 2, R}\left(x_{j-1 / 2}\right)\right)=\hat{u}_{j-1 / 2, L}\left(x_{j-1 / 2}\right), \\
& u_{j+1 / 2}=u\left(\hat{u}_{j+1 / 2, L}\left(x_{j+1 / 2}\right), \hat{u}_{j+1 / 2, R}\left(x_{j+1 / 2}\right)\right)=\hat{u}_{j+1 / 2, L}\left(x_{j+1 / 2}\right) .
\end{aligned}
$$

The recovered function $f$ is substituted for any $u$-quantities of the diffusion boundary terms,

$$
\left[v_{j} \partial_{x} u_{j}-u_{j} \partial_{x} v_{j}\right]_{x_{j-1 / 2}}^{x_{j+1 / 2}}=\left[v_{j} \partial_{x} f-f \partial_{x} v_{j}\right]_{x_{j-1 / 2}}^{x_{j+1 / 2}}
$$

The original discrete solution $u_{j}(x)$ is used in both advection and diffusion volume integrals, $\int_{\Omega_{j}} u_{j} \partial_{x} v_{j} d x$ and $\int_{\Omega_{j}} u_{j} \partial_{x x} v_{j} d x$ respectively.

Equation (22) is then rewritten into the following matrix form:

$$
\frac{\Delta x}{a} \frac{d}{d t} \mathbf{a}_{j}=\underbrace{\left[\mathbf{M}(T)_{\mathrm{advec}}+\frac{1}{P e} \mathbf{M}(T)_{\mathrm{diff}}\right]}_{\mathbf{M}(T)} \cdot \mathbf{a}_{j} ;
$$



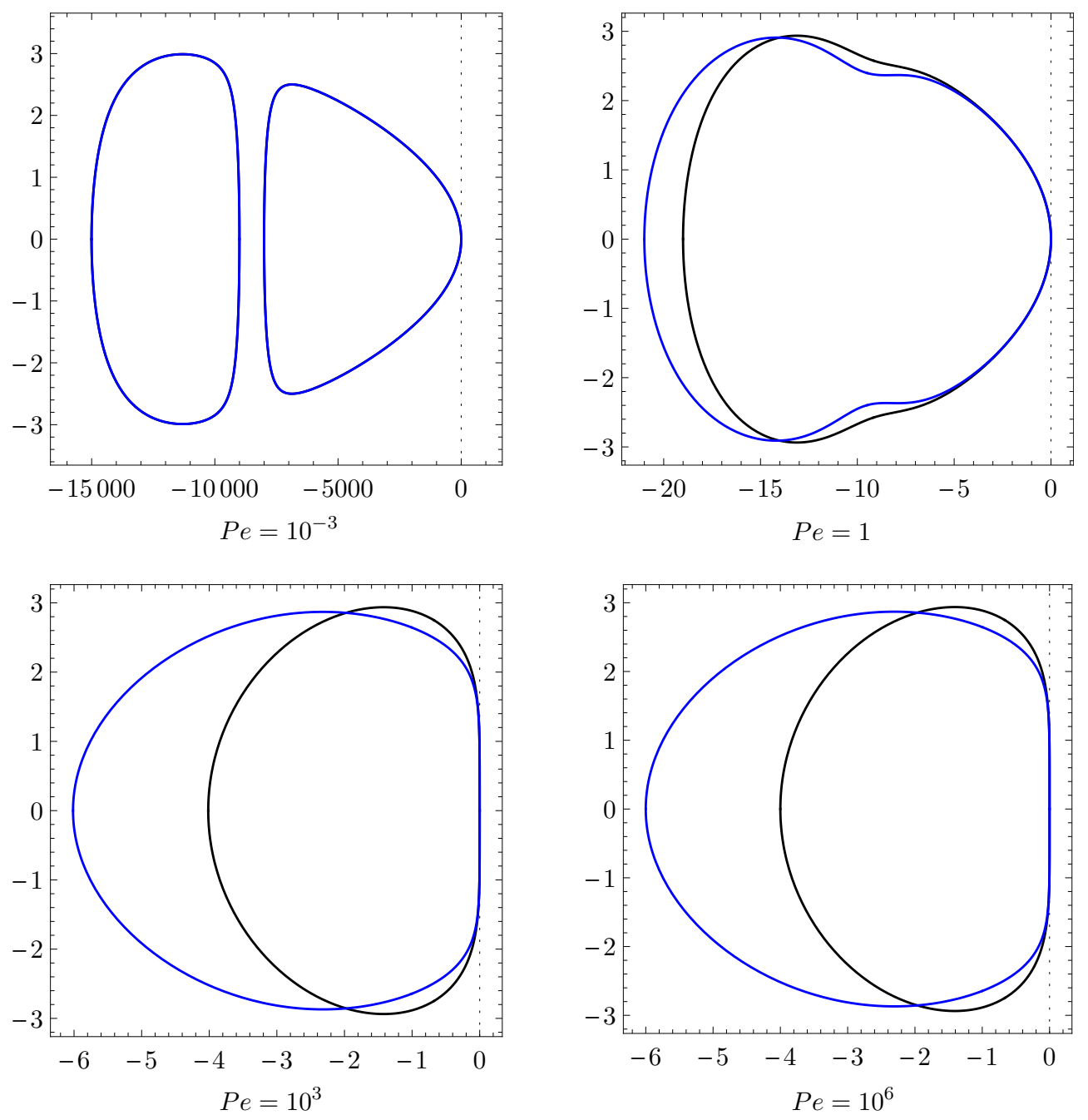

icb1 $[0]$

icb1 $[1]$

Figure 3: Eigenvalues of the combined schemes of RDG-2x and icb1[\#] $(p=1)$ at various values of Pe number. Note that the legend indicates only the specific advection discretization that is combined with RDG.

here $T$ represents forward translation by one cell, $T a_{j}^{(\alpha)}=a_{j+1}^{(\alpha)}$ and $T^{-1} a_{j}^{(\alpha)}=a_{j-1}^{(\alpha)} ; P e$ is the cell Peclet number, $P e=a \Delta x / \mu$. We determine the Fourier transform of the matrix operator $\mathbf{M}(T)$, $\widehat{\mathbf{M}}(\beta)$, by substituting $T$ for its Fourier transform $e^{i \beta}$. Then the eigenvalues of $\widehat{\mathbf{M}}(\beta)$ are calculated and their Taylor-series expansions are compared against the exact differential operator in Fourier mode, which is

$$
\lambda_{\text {exact }}=-i \beta-\frac{1}{P e} \beta^{2} .
$$

The order of accuracy and stability properties of the overall scheme will then be determined.

\section{III.B. Results}

The objectives of our analyses are a) to validate the order of accuracy of the combination of RDG and various icb schemes, especially the optimal variations (icb1[\#], icb2[\#, \#], and icb3[\#,\#,\#]); and b) to examine the effect of adding the diffusion term on the stability of icb advection discretization. 

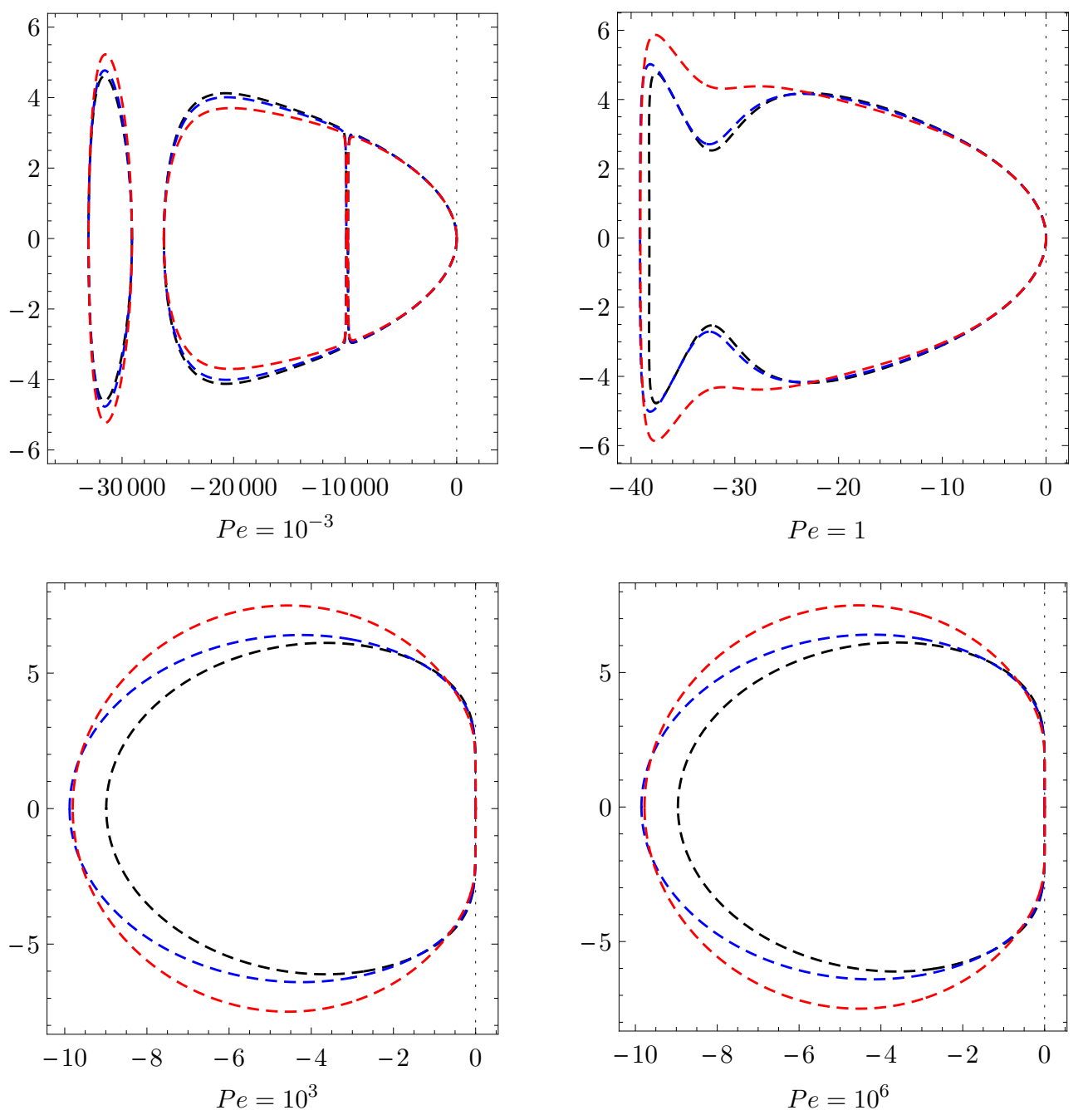

$----i c b 2[0] \quad----i c b 2[1]----i c b 2[2]$

Figure 4: Eigenvalues of the combined schemes of RDG-2x and icb2[\#] $(p=2)$ at various values of Pe number. Note that the legend indicates only the specific advection discretization that is combined with RDG.

At $p=1$, the icb1[\#] and RDG discretizations have the same fourth order of accuracy, when used separately for advection and diffusion problems. The analysis shows that, when they are combined to discretize the advection-diffusion problem, the order of accuracy is preserved. Figure 3 displays the eigenvalue loci in the complex plane at various values of the $P e$ number; their real parts are always negative, indicating a favorable stability property.

For $p=2$, the eigenvalue loci of the combinations of RDG and icb2 [\#] or icb2 [\#,\#] are shown in Figs. 4 and 5, respectively. The overall orders of accuracy are confirmed to be sixth and seventh, same as those of the advection discretizations as shown in Table 1. Our previous analyses $^{[25]}$ showed that the eigenvalues of icb2 [\#] schemes have non-positive real parts, which stays unchanged when combined with RDG. For icb2 [\#,\#] schemes, at the advection limit, the real parts of the eigenvalues are positive for a certain range of $\beta$, although the magnitude is quite small (in the order of $10^{-4}$ ), as shown in Fig. 6. Indeed our results at $P e=10^{6}$ have positive real parts but they are too small to appear on the current plotting scale, while those of $P e=10^{3}$ shows no sign of positive real parts. Therefore, the "transitional" value for $P e$ number lies between $10^{3}$ and $10^{6}$. 

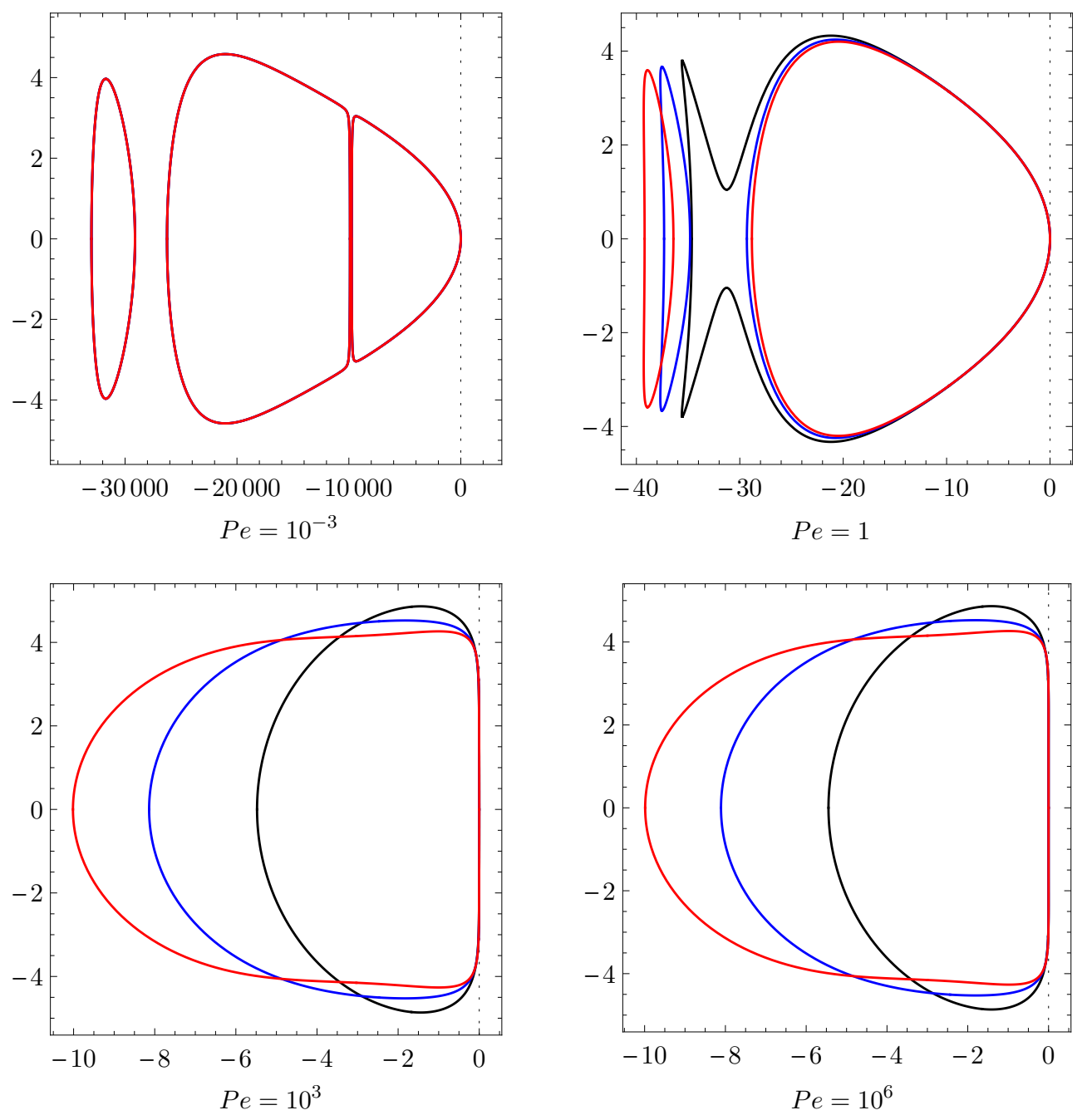

icb2 $[0,1]$

icb2 $[0,2]$

$i \operatorname{cb} 2[0,3]$

Figure 5: Eigenvalues of the combined schemes of RDG-2x and icb2[\#,\#] $(p=2)$ at various values of Pe number. Note that the legend indicates only the specific advection discretization that is combined with RDG.

- For icb2 $[0,1]$, the transitional value is $P e \approx 6000$,

- for icb2 [0,2], it is $P e \approx 9000$, and

- for icb2 [1,2], it happens at $P e \approx 11000$.

This behavior is consistent with our observation at the advection limit, at which the incursion into the positive half-plane of $i c b 2[1,2]$ is less than those of $i c b 2[0,1]$ and $i c b 2[0,2]$. It is therefore preferable to preserve the higher-order moments of the other cells, which means we choose the option in which $\mathcal{K}$ contains indices of the higher-order moments.

Behaviors at $p=3$ are similar to the $p=2$ case, except that the combination of RDG and icb3 [\#,\#,\#] has smaller value for the transitional $P e$ number: $\approx 2000$ for the combination with icb3 $[0,1,2], \approx 3000$ with icb3 [0,1,3], $\approx 4000$ with icb3 [0,2,3], and $\approx 4500$ for the combination with icb3 $[1,2,3]$. Their eigenvalue loci are shown in Figs. 7 to 10; and the orders of accuracy are confirmed as eighth for the icb3[\#] combinations, ninth for the icb3[\#,\#] combinations, and tenth for the $i \mathrm{cb} 3[\#, \#, \#]$ combinations. 


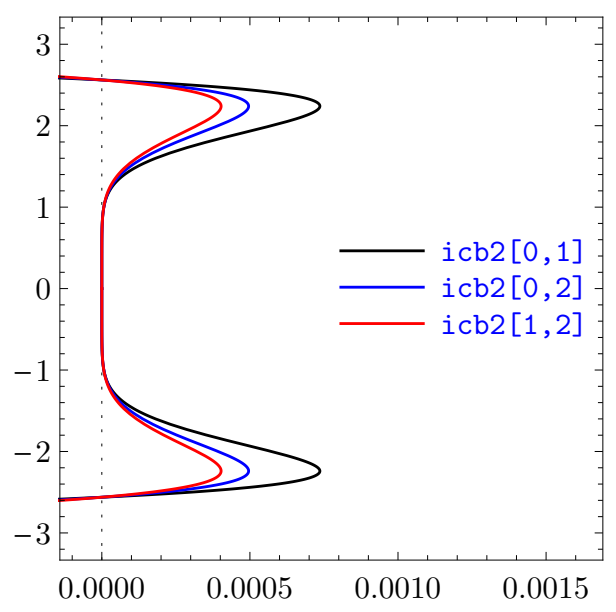

Figure 6: Zoom into the region around vertical axis in Fig. 5 to show the unstable domains of the icb2 [\#, \#] eigenvalues.

\section{Conclusions and future developments}

We have presented a DG discretization for the advection-diffusion equation that combine Recovery-based DG (RDG) for the diffusion terms and interface-centered binary-reconstruction DG for advection (icb). The latter is our preferred approach to improve upon the standard upwind DG for advection on a compact stencil.

Results from Fourier analysis are displayed for $p \in[1,3]$. They confirm that the aforementioned combination indeed achieves the predicted order $3 p+1$.

In the fist case of $p=1$, the real components of the eigenvalues are strictly non-positive for all values of $P e$ number, implying good stability properties. In the second case of $p=2$, the eigenvalues go slightly into the positive half-plane when the advection limit is approached. The transitional values is 6000, 9000, and 11000 for the $i c b 2[0,1]$, icb2 [0,2], and icb2 [1,2] schemes, respectively. It shows that we it is preferable to use a icb scheme of which $\mathcal{K}$ contains higher-order moments. Switching from $\mathcal{K}=\{0,1\}$ to $\mathcal{K}=\{1,2\}$ almost doubles the Peclet range that its eigenvalues stay strictly on the negative plane. At $p=3$, the schemes behave similarly, except that the transitional values for the combination of RDG and icb3[\#,\#,\#] are even smaller.

Small transitional values of the Peclet number show that physical dissipation alone is not strong enough to fully stabilize the icb schemes. Our next step is to study the dissipative errors of these schemes in order to check if it is feasible to add artificial dissipation to these schemes. 

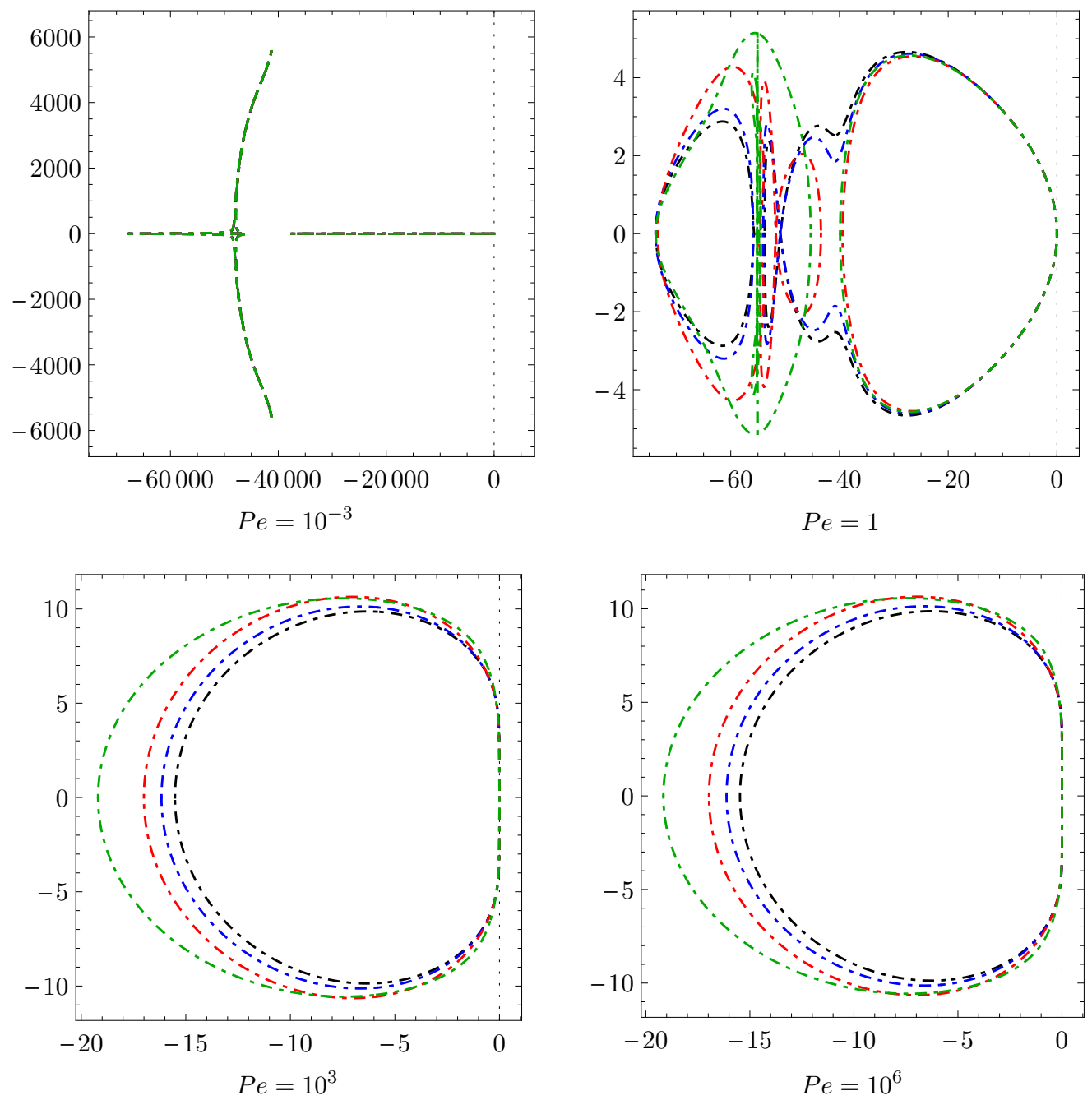

$$
\text { - - - icb3 }[0] \quad--\cdot-\text { icb3 }[1]
$$

- - - icb3[2] - - - - icb3[3]

Figure 7: Eigenvalues of the combined schemes of RDG-2x and icb3[\#] $(p=3)$ at various values of Pe number. Note that the legend indicates only the specific advection discretization that is combined with RDG. 

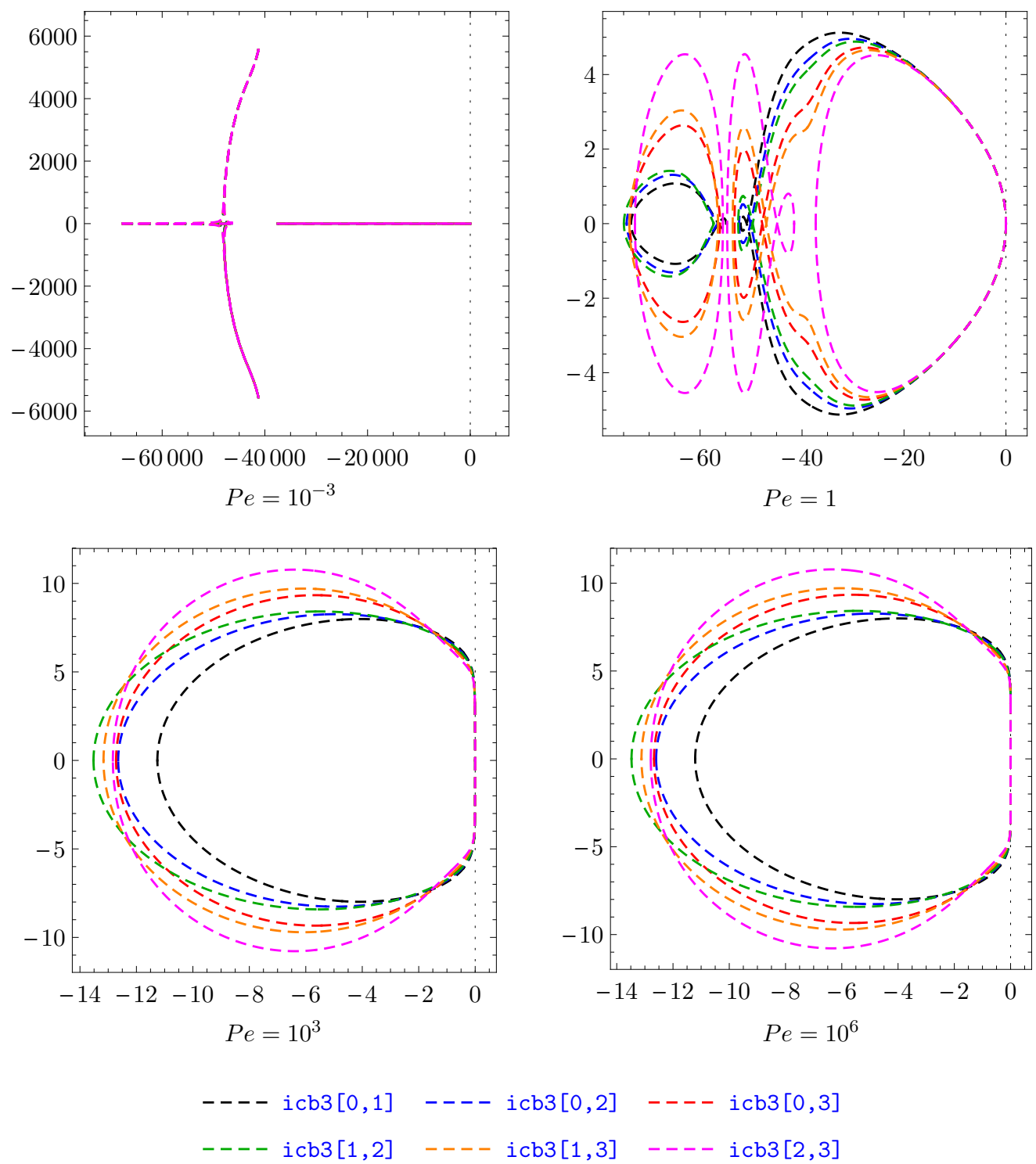

Figure 8: Eigenvalues of the combined schemes of RDG-2x and icb3[\#,\#] $(p=3)$ at various values of $P e$ number. Note that the legend indicates only the specific advection discretization that is combined with RDG. 

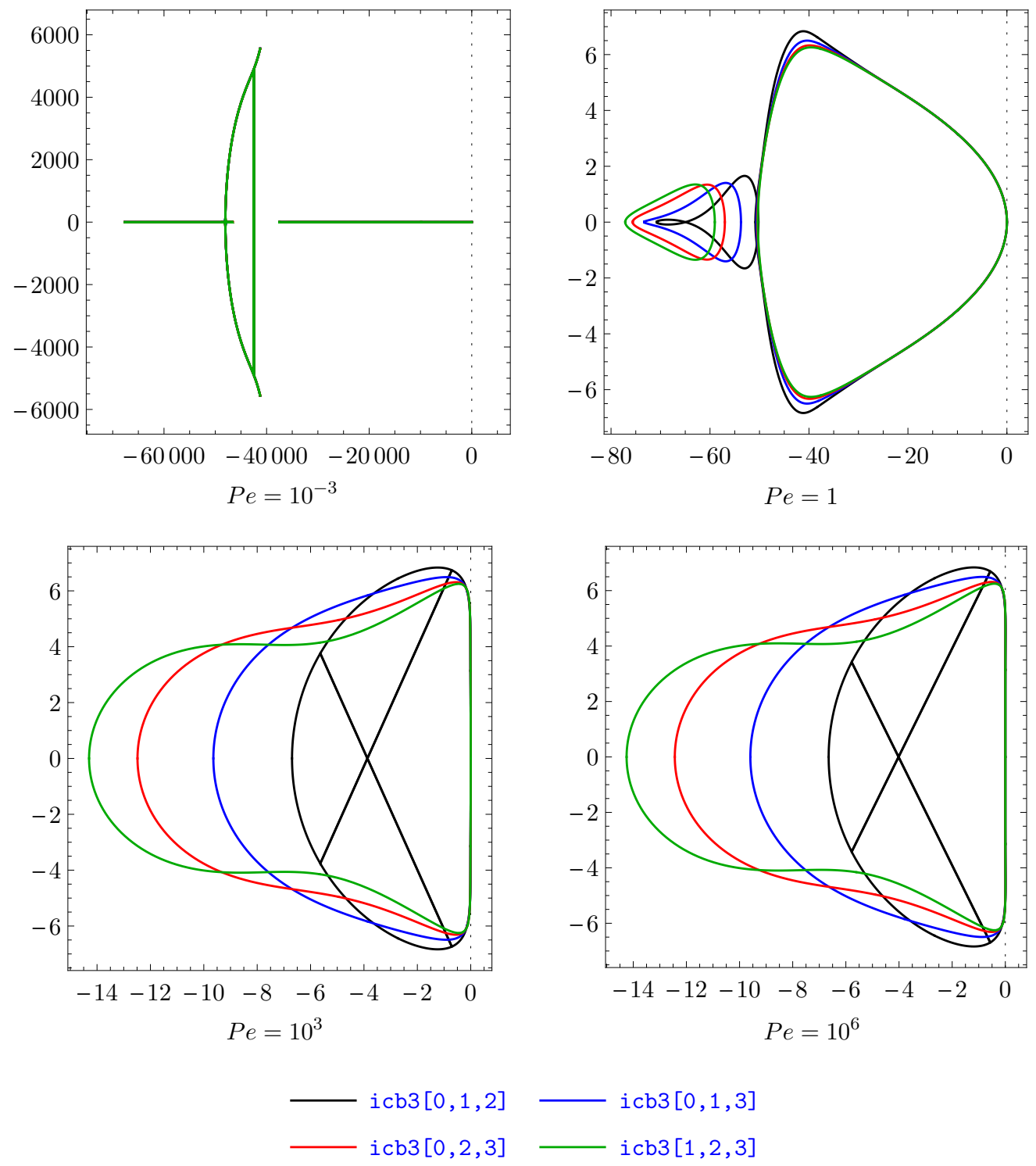

Figure 9: Eigenvalues of the combined schemes of RDG-2x and icb3[\#,\#,\#] $(p=3)$ at various values of $P e$ number. Note that the legend indicates only the specific advection discretization that is combined with RDG; and the straight-line segments in the eigenvalue loci of icb3 $[0,1,2]$ at $P e=10^{3}$ and $P e=10^{6}$ are the plotting artifacts. 

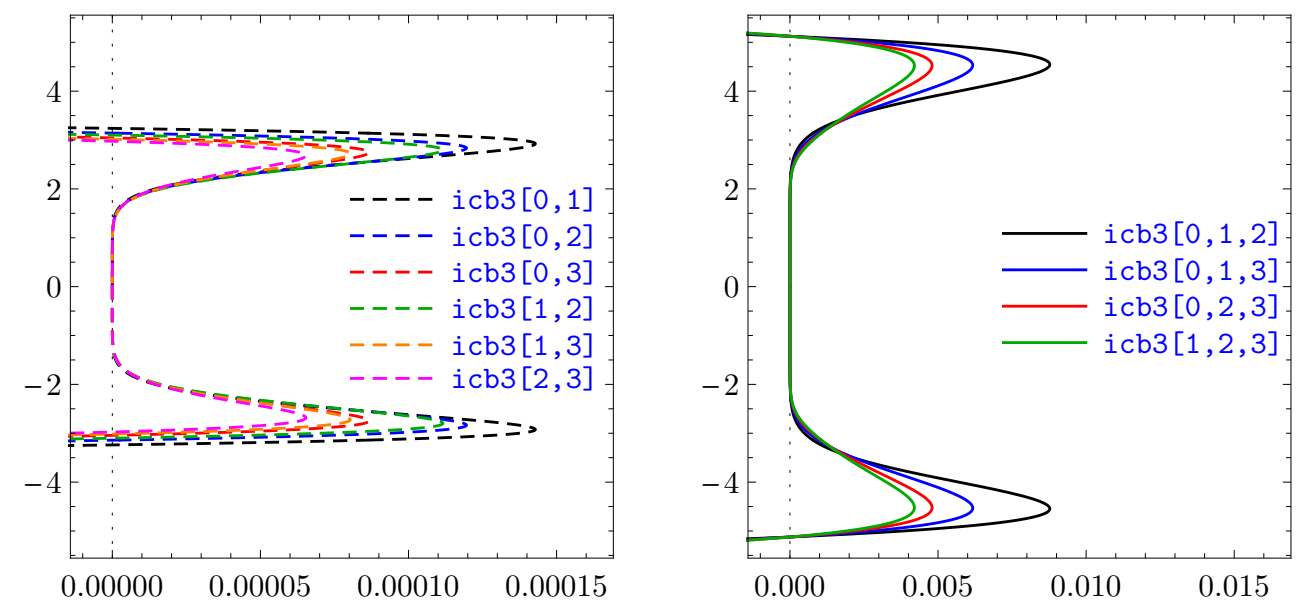

Figure 10: Zoom into the region around vertical axis in Figs. 8 and 9 to show the unstable domains of the icb3[\#,\#] and icb3 [\#, \#, \#] eigenvalues. 


\section{References}

1 W.H. Reed and T.R. Hill. Triangular mesh methods for the neutron transport equation. Technical Report LA-UR-73-479, Los Alamos Scientific Laboratory, 1973.

2 Claes Johnson and Juhani Pitkäranta. An analysis of the discontinuous Galerkin method for a scalar hyperbolic equation. Mathematics of Computation, 46(173):1-26, 1986.

3 P LeSaint and Pierre-Arnaud Raviart. On a finite element method for solving the neutron transport equation. In C. De Boor, editor, Mathematical aspects of finite elements in partial differential equations, page 89. Academic Press, 1974.

4 Todd E Peterson. A note on the convergence of the discontinuous Galerkin method for a scalar hyperbolic equation. SIAM Journal on Numerical Analysis, 28(1):133-140, 1991.

5 Gerard R Richter. An optimal-order error estimate for the discontinuous Galerkin method. Mathematics of Computation, 50(181):75-88, 1988.

${ }^{6}$ Bernardo Cockburn and Chi-Wang Shu. TVB runge-kutta local projection discontinuous Galerkin finite element method for conservation laws. II. General framework. Mathematics of Computation, 52(186): 411-435, 1989.

7 Bernardo Cockburn, San-Yih Lin, and Chi-Wang Shu. TVB runge-kutta local projection discontinuous Galerkin finite element method for conservation laws III: One-dimensional systems. Journal of Computational Physics, 84(1):90-113, 1989.

8 Bernardo Cockburn, Suchung Hou, and Chi-Wang Shu. The runge-kutta local projection discontinuous Galerkin finite element method for conservation laws. IV. The multidimensional case. Mathematics of Computation, 54(190):545-581, 1990.

9 Bernardo Cockburn and Chi-Wang Shu. The rungekutta discontinuous Galerkin method for conservation laws V: Multidimensional systems. Journal of Computational Physics, 141(2):199-224, 1998.

10 D. N. Arnold, F. Brezzi, B. Cockburn, and L. D. Marini. Unified analysis of discontinuous Galerkin methods for elliptic problems. SIAM Journal on Numerical Analysis, 39(5):1749-1779, 2002.

11 F. Brezzi, G. Manzini, D. Marini, P. Pietra, and A. Russo. Discontinuous Galerkin approximations for elliptic problems. Numerical Methods for Partial Differential Equations, 16(4):365-378, 2000.

12 Jim Douglas and Todd Dupont. Interior penalty procedures for elliptic and parabolic Galerkin methods. In Computing methods in applied sciences, pages 207-216. Springer, 1976.

13 F. Bassi and S. Rebay. A high-order accurate discontinuous finite element method for the numerical solution of the compressible Navier-Stokes equations. Journal of Computational Physics, 131(2):267-279, 1997.

14 F Bassi, S Rebay, G Mariotti, S Pedinotti, and M Savini. A high-order accurate discontinuous finite element method for inviscid and viscous turbomachinery flows. In Proceedings of 2nd European Conference on Turbomachinery, Fluid Dynamics and Thermodynamics, pages 99-108, Technologisch Instituut, Antwerpen, Belgium, 1997.

15 B. Cockburn and C. W. Shu. The local discontinuous Galerkin method for time-dependent convectiondiffusion systems. SIAM Journal on Numerical Analysis, 35(6):2440-2463, 1998.

16 J. Peraire and P. O. Persson. The compact discontinuous Galerkin (CDG) method for elliptic problems. Siam Journal on Scientific Computing, 30(4):1806-1824, 2008.

17 Bram Van Leer and Shohei Nomura. Discontinuous Galerkin for diffusion. In 17th AIAA Computational Fluid Dynamics Conference, Toronto, Ontario, Canada, 6-9 June 2005. American Institute of Aeronautics and Astronautics. AIAA 2005-5108. 
18 Marcus Lo and Bram Van Leer. Analysis and implementation of recovery-based discontinuous Galerkin for diffusion. In 19th AIAA Computational Fluid Dynamics Conference, San Antonio, Texas, US, 22-25 June 2009. American Institute of Aeronautics and Astronautics. AIAA 2009-3786.

19 Hung T. Huynh. A reconstruction approach to high-order schemes including discontinuous Galerkin for diffusion. In 47th AIAA Aerospace Sciences Meeting Including The New Horizons Forum and Aerospace Exposition, Orlando, Florida, US, 5-8 January 2009. American Institute of Aeronautics and Astronautics. AIAA 2009-0403.

20 Kwok Ho Marcus Lo. A space-time discontinuous Galerkin method for Navier-Stokes with recovery. $\mathrm{PhD}$ thesis, The University of Michigan, 2011.

21 Marcus Lo and Bram Van Leer. Recovery-based discontinuous Galerkin for Navier-Stokes viscous terms. In 20th AIAA Computational Fluid Dynamics Conference, Honolulu, Hawaii, US, 27-30 June 2011. American Institute of Aeronautics and Astronautics. AIAA 2011-3406.

22 Eric Johnsen, Sreenivas Varadan, and Bram Van Leer. A three-dimensional recovery-based discontinuous Galerkin method for turbulence simulations. In 51st AIAA Aerospace Sciences Meeting, Grapevine, Texas, US, 7-10 January 2013. American Institute of Aeronautics and Astronautics. AIAA 2013-0515.

23 Eric Johnsen, Sreenivas Varadan, and Aditya Nair. Recovery discontinuous Galerkin method for compressible turbulence. In 21st AIAA Computational Fluid Dynamics Conference, San Diego, California, US, 24-27 June 2013. American Institute of Aeronautics and Astronautics. AIAA 2013-3066.

24 Eric Johnsen, Loc Khieu, and Aditya Nair. A simple method to improve the accuracy of advection in discontinuous Galerkin methods for Navier-Stokes simulations. In 52nd Aerospace Science Meeting, National Harbor, Maryland, US, 13-17 January 2014. American Institute of Aeronautics and Astronautics. AIAA 2014-1276.

25 Loc Khieu and Eric Johnsen. Analysis of improved advection schemes for discontinuous Galerkin methods. In 44th AIAA Fluid Dynamics Conference, Atlanta, Georgia, US, 16-20 June 2014. American Institute of Aeronautics and Astronautics. AIAA 2014-3221.

26 Michael Dumbser. Arbitrary high order schemes for the solution of hyperbolic conservation laws in complex domains. Shaker, 2005.

27 M Dumbser and C. D. Munz. Arbitrary high order discontinuous Galerkin schemes. In Stéphane Cordier, Thierry Goudon, Michaël Gutnic, and Eric Sonnendrücker, editors, Numerical methods for hyperbolic and kinetic problems, volume 7 of IRMA Lectures in Mathematics and Theoretical Physics, pages 295-333. EMS Publishing House, 2005.

28 M. Dumbser, D. S. Balsara, E. F. Toro, and C. D. Munz. A unified framework for the construction of onestep finite volume and discontinuous Galerkin schemes on unstructured meshes. Journal of Computational Physics, 227(18):8209-8253, 2008. 